\title{
A time-resolved imaging and electrical study on a high current atmospheric pressure spark discharge
}

\author{
J. M. Palomares, ${ }^{1,2, a)}$ A. Kohut, ${ }^{3}$ G. Galbács, ${ }^{4}$ R. Engeln, ${ }^{1}$ and Zs. Geretovszky ${ }^{3}$ \\ ${ }^{1}$ Department of Applied Physics, Eindhoven University of Technology, Eindhoven $5600 \mathrm{MB}$, The Netherlands \\ ${ }^{2}$ Dutch Institute for Fundamental Energy Research (DIFFER), PO Box 6336, 5600 HH Eindhoven, \\ The Netherlands \\ ${ }^{3}$ Department of Optics and Quantum Electronics, University of Szeged, Szeged H-6720, Hungary \\ ${ }^{4}$ Department of Inorganic and Analytical Chemistry, University of Szeged, Szeged H-6720, Hungary
}

(Received 8 October 2015; accepted 1 December 2015; published online 21 December 2015)

\begin{abstract}
We present a time-resolved imaging and electrical study of an atmospheric pressure spark discharge. The conditions of the present study are those used for nanoparticle generation in spark discharge generator setups. The oscillatory bipolar spark discharge was generated between two identical $\mathrm{Cu}$ electrodes in different configurations (cylindrical flat-end or tipped-end geometries, electrode gap from 0.5 to $4 \mathrm{~mm}$ ), in a controlled co-axial $\mathrm{N}_{2}$ flow, and was supplied by a high voltage capacitor. Imaging data with nanosecond time resolution were collected using an intensified CCD camera. This data were used to study the time evolution of plasma morphology, total light emission intensity, and the rate of plasma expansion. High voltage and high current probes were employed to collect electrical data about the discharge. The electrical data recorded allowed, among others, the calculation of the equivalent resistance and inductance of the circuit, estimations for the energy dissipated in the spark gap. By combining imaging and electrical data, observations could be made about the correlation of the evolution of total emitted light and the dissipated power. It was also observed that the distribution of light emission of the plasma in the spark gap is uneven, as it exhibits a "hot spot" with an oscillating position in the axial direction, in correlation with the high voltage waveform. The initial expansion rate of the cylindrical plasma front was found to be supersonic; thus, the discharge releases a strong shockwave. Finally, the results on equivalent resistance and channel expansion are comparable to those of unipolar arcs. This shows the spark discharge has a similar behavior to the arc regime during the conductive phase and until the current oscillations stop. @ 2015 AIP Publishing LLC. [http://dx.doi.org/10.1063/1.4937729]
\end{abstract}

\section{INTRODUCTION}

Spark discharge generators (SDG) used for nanoparticle (NP) generation employ a high voltage electrical discharge operated in a controlled gas environment (usually an inert gas at around atmospheric pressure) between two conducting electrodes (metals or carbon) of identical, mostly cylindrical shape. The combined heating and sputtering effect of the high temperature and high current spark plasma filament causes the erosion of the electrode material, which will then condensate and aggregate to form NPs. Recently, the application of spark discharges for NP production has been steadily increasing, as it was realized that SDGs provide an exceptionally versatile, clean, and efficient method of NP production. Major contributions to the advancement of the field came from Schmidt-Ott, ${ }^{1}$ as well as from Hwang, ${ }^{2}$ Deppert, ${ }^{3}$ and Kruis. ${ }^{4}$ The most recent review of the area was published in Ref. 1. It is also worth mentioning that pulsed or condensed arc discharges can also be used to generate nanomaterials. ${ }^{5-7}$

It is an interesting fact that in spite of the extensive use of SDGs for NP production, still only a small fraction of the fundamental physical processes occurring in the SDG are fully understood. Only a few detailed studies can be found in

${ }^{a)}$ Email: j.m.p.linares@differ.nl literature attempting to describe the NP generation process in spark discharges. ${ }^{2,8-10}$ In our view, one of the key reasons for this is that most of the studies being published on SDGs are conducted with an engineering application in mind. Another point is that the appealing simplicity of the technical realization of NP production is hiding away the very complex nature of the underlying physical and chemical processes. At the same time, the physics studies in the literature on spark discharges running at atmospheric pressure concentrate on fields like gas treatment and plasma assisted combustion. ${ }^{11-15}$ These applications require short and medium intensity current pulses $(<100 \mathrm{~ns}$, some tens of amps, respectively) in order to achieve an efficient and rapid release of heat and radicals while avoiding damage to the electrodes. As opposed to this, NP generation in an SDG aims for electrode erosion, thus requires long duration, high intensity current pulses (ca. $10 \mu \mathrm{s}$, from hundreds to thousands of amps, respectively).

Fast imaging is a reliable tool to study the breakdown in gases under different conditions. ${ }^{16-21}$ Most of the discharge imaging studies either focus on streamer formation and propagation, or globally analyze the different discharge regimes such as glow, corona, or spark. In the present study, we focus on how the spark channel evolves once it is formed, always under the conditions of $\mu$ s-long and high intensity current peaks required for NP formation. We follow the temporal 
evolution of the plasma morphology, position, intensity, and size up until a few tens of microseconds. The particular SDG setup used in the present work has been already used for NP generation under identical experimental conditions, ${ }^{4}$ and although no simultaneous particle data have been collected this time, the results of this study are directly relevant for the understanding of NP synthesis and electrode erosion processes.

Initiated by the lack of fundamental physical data available in the literature for the conditions of atmospheric pressure NP generation by spark discharges, the aim of the present work was to collect systematic electrical and time resolved imaging data on the operation of an SDG under the influence of $\mu$ s-long and high current peaks required for electrode erosion and hence NP formation. The electric monitoring combined with the plasma morphological data obtained by imaging measurements provide important information on the plasma movement and size, and the morphological evolution of the plasma channel. These quantities are measured as a function of external parameters most relevant for NP production, such as the electrode distance, geometry, and gas flow rate.

\section{EXPERIMENTAL SETUP}

\section{A. The spark discharge generator}

A schematic view of the experimental setup can be seen in Fig. 1. The central parts of all SDG's are the spark chamber and the electrical power circuit. ${ }^{3}$ In the present study, a KFsealed, DN-160 sized, cylindrical stainless steel chamber was used, equipped with 6 pieces of radially located KF-40 ports. One of the KF-160 ports was sealed by a fused silica window to facilitate optical observation. The $\mathrm{Cu}$ electrodes $(99.9 \% \mathrm{pu}-$ rity) were vertically aligned and an upward directed, co-axial $\mathrm{N}_{2}$ carrier gas flow was employed during NP generation. This chamber geometry has been developed for the purposes of the BUONAPART-E project, ${ }^{22}$ supported by the 7 th Framework Programme of the European Union, specifically optimized for
NP production purposes, and was manufactured by Pfeiffer Vacuum GmbH. The distance between the electrodes (i.e., the size of the electrode gap) was controlled by micrometer screws in the range from 0.5 to $4.0 \mathrm{~mm}$. The flow rate of the $99.995 \%$ purity nitrogen gas was set by a mass flow controller (Model F-201CV-10k, Bronkhorst). The flow rate was kept constant at $10 \mathrm{slm}$ in all experiments, except those aiming to study the effect of flow rate. All experiments were carried out at atmospheric pressure.

Cylindrical copper electrodes of $6.35 \mathrm{~mm}$ diameter were used in two electrode geometries: flat-end and tipped-end (with a $30^{\circ}$ apex angle). In the flat-end geometry, electrode erosion is distributed over a larger area (i.e., the entire front surface of the electrode rods), while on tipped-end electrodes, erosion is concentrated on the electrode tips. Since efficient NP generation needs substantial electrode erosion the flat-end geometry is preferred in SDGs. However, with this electrode geometry, the wandering of the sparks over the electrode surface poses challenges to optical measurements and the electrical data are less stable due to positional variation of the surface quality and the concomitant differences in the breakdown voltage. Hence results obtained with tippedend electrodes provide more reliable information, and more fundamental insight, while the observations obtained with flat-end electrode has more direct relevance to NP production. The flat-end geometry also requires a higher breakdown voltage for identical conditions, due to the absence of the high electrical field at the tip of tipped-end electrodes. A practical consequence of this is that the maximum output voltage of $10 \mathrm{kV}$ of our power supply restricted us to the use of electrode gaps not larger than $2 \mathrm{~mm}$ with flat-end electrodes. The effect of erosion on the inter-electrode distance is balanced by monitoring the breakdown voltage, and the gap size was frequently fine-adjusted to keep the breakdown voltage constant during the experiments.

Energy is pumped into the spark gap by a simple capacitor charging circuit. A capacitor bank (referred to as

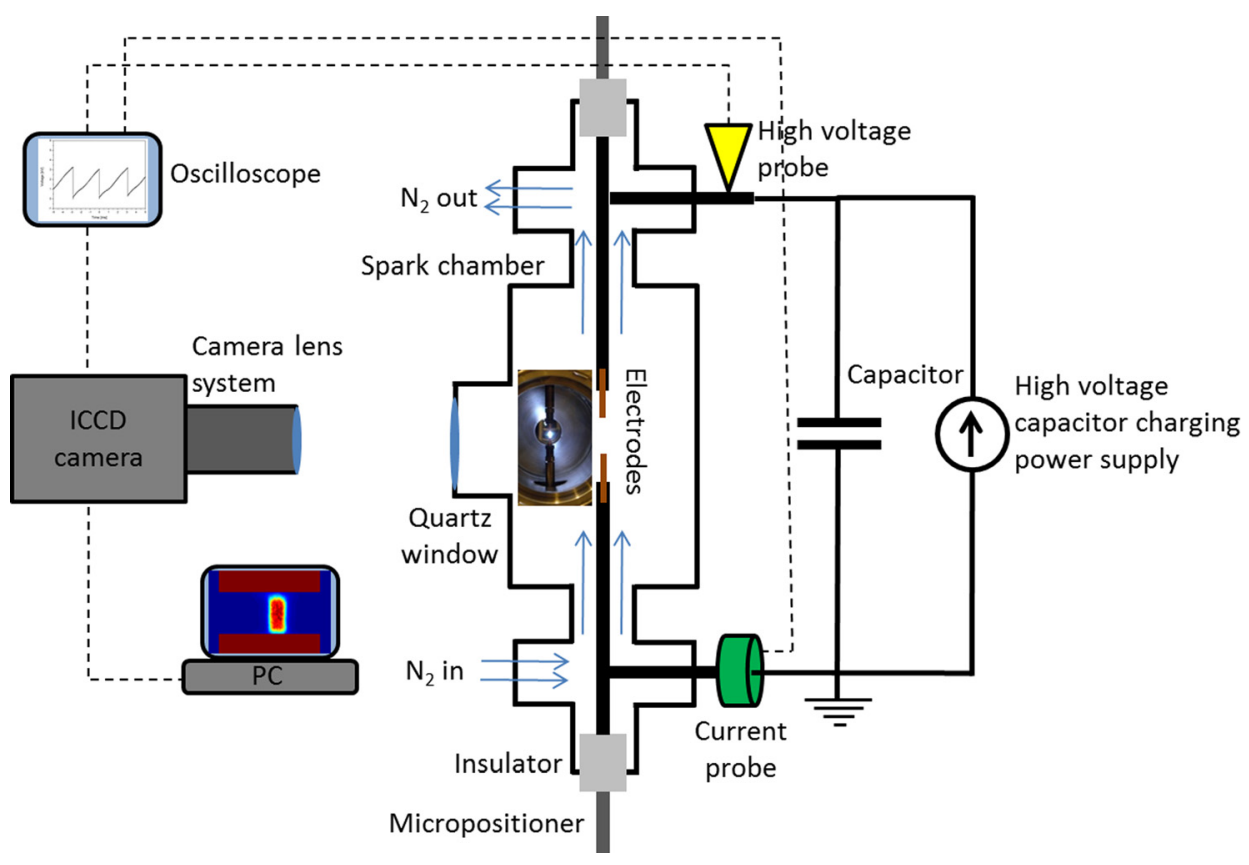

FIG. 1. Schematic view of the experimental setup. For the sake of clarity, only the used ports of the chamber are shown. 
capacitor in the rest of this paper) with a total capacitance of $29 \mathrm{nF}$ is charged by a high voltage capacitor charger power supply (Model CCR10-P-750, Technix) to a maximum voltage of $10 \mathrm{kV}$ using a charging current of max. $150 \mathrm{~mA}$. Since the capacitor is directly connected to the electrode gap (Fig. 1), the discharge of the capacitor across the spark gap will commence when the voltage on the capacitor reaches the breakdown voltage of the gas in the spark gap. The resulting spark discharge is a bipolar, oscillatory discharge, the damping, and the oscillation frequency of which is set by the total capacitance, resistance, and inductance of the discharge circuit. The discharge of the capacitor via the spark gap is accompanied by the recharging of the capacitor by the charging power supply ("self-pulsed, free running mode"), but since the current in the charging loop is many orders of magnitude smaller than the current in the discharging loop, the two processes can be considered to be practically independent. The spark repetition rate (SRR) can be controlled by changing the charging current and the gap size (via the breakdown voltage), assuming that all other experimental conditions are kept constant. The voltage and current waveforms in the discharge loop were recorded on a digital storage oscilloscope (Model DSOX2024A, Agilent, $200 \mathrm{MHz}$ ) using electrical probes: a high voltage probe (Model N2771B, Agilent, $50 \mathrm{MHz}$ ) and a calibrated current probe (Model 110, Pearsons, $20 \mathrm{MHz}$ ). In terms of time response, the limiting component is the current probe, with a bandwidth of $20 \mathrm{MHz}$ and a shortest measurable rise time of $20 \mathrm{~ns}$. Although this probe is not suitable for measuring transient features, such as the characteristics of the streamer preceding the breakdown, it can perfectly measure the current oscillations occurring in the post-breakdown phase that is the interest of the present work. It is also important that this current probe is capable of handling the peak currents of $450 \mathrm{~A}$ typical in our spark discharge, for $\sim 1 \mu$ s or longer times.

A typical high voltage waveform acquired on repetitive spark events is shown in Fig. 2. The voltage signal over several spark events is plotted for the particular case of

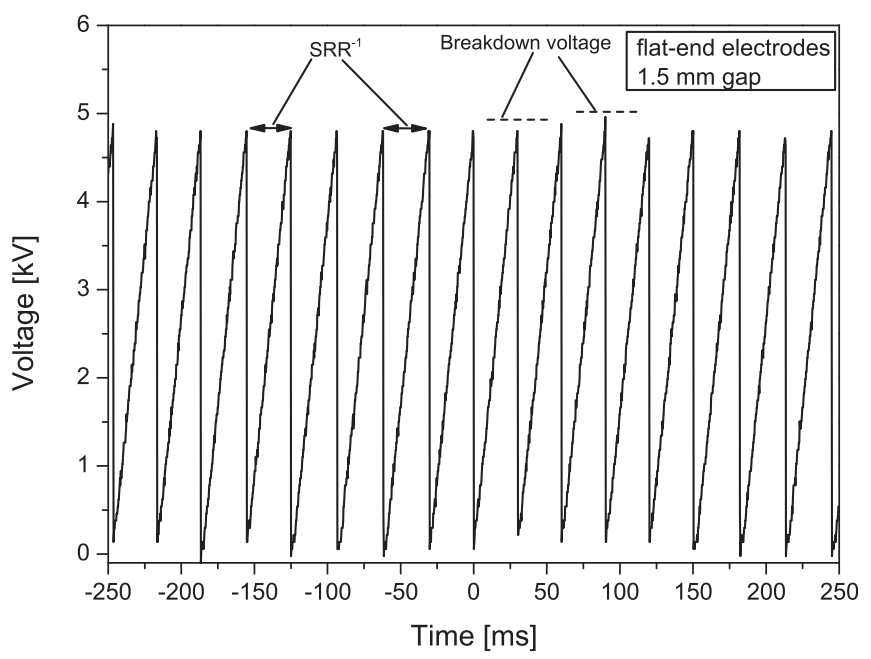

FIG. 2. Voltage signal measured for consecutive spark events recorded between flat electrodes with $1.5 \mathrm{~mm}$ gap. The peak values represent the breakdown voltage for the given spark. The inverse of the duration between consecutive peaks gives the (repetition) frequency of the spark, i.e., the SRR. cylindrical flat-end electrodes separated by a $1.5 \mathrm{~mm}$ gap. Values for the average breakdown voltage, the SRR, as well as their scatter were obtained from such measurements. During the present experiments, the repetition rate of the spark was kept at $35 \pm 1 \mathrm{~Hz}$ under all conditions.

Based on data taken from many consecutive sparks (Fig. 2), we estimated the precision of the breakdown voltage to be ca. $5 \%$, which results in a ca. $10 \%$ relative error in the calculated energy stored in the capacitor. Similarly, the precision of the peak current was found to be ca. $2.5 \%$, and thus, the calculated resistance value had a ca. $2.5 \%$ relative error. The combination of these values gives rise to a $5.5 \%$ relative error in the measured spark energy (see later). These error values only include the scatter of the electrical data and do not include the accuracy of our voltage and current probes $(<2 \%)$ as well as the scatter of the actual capacitance values of the used capacitors ( $\sim 5 \%$ nominal).

\section{B. Time resolved fast imaging}

Time-resolved images of the spark were recorded using a high sensitivity, ns-gated intensified CCD (ICCD) camera (Model iStar-734 Gen 2 (W-AGT, -03), Andor), with a photographic lens system $(\mathrm{WD}=2000 \mathrm{~mm}$ ) attached to it. For triggering the ICCD camera, the falling edge of the voltage signal measured by the high voltage probe was used. Due to the insertion delay of the camera and the delay caused by signal propagation, the minimum total delay of recording imaging data was $50 \mathrm{~ns}$. In order to study the time evolution of the spark, images of the discharge were taken at time delays varying from $50 \mathrm{~ns}$ to a few $\mathrm{ms}$. The gate width of the camera was progressively varied between $5 \mathrm{~ns}$ and $100 \mu \mathrm{s}$ in order to compensate for the decreasing emitted light intensity at longer delays times. The general conditions, such as electrode geometry, gap length, spark repetition rate, and breakdown voltage were kept constant. Correction for different gate widths, amplification, and background was carried out by post-processing the images using a MATLAB code. The sensitivity of the camera and the transmission curve of the photographic lens allowed taking wavelength-integrated intensity data in the $200-850 \mathrm{~nm}$ range.

\section{RESULTS}

\section{A. Electrical results}

In Fig. 3, typical voltage and current waveforms are shown for tipped electrodes placed $3 \mathrm{~mm}$ apart. The underdamped character of the oscillations is immediately obvious. As it can be seen, the voltage drops abruptly and then it oscillates with exponentially decaying amplitude, while the current rises fast and oscillates in a similar manner, but with a certain phase shift with respect to the voltage signal. Under the conditions used in this study, decay times (defined as the inverse of the damping coefficient of the current or voltage oscillations) in the range of $10-15 \mu$ s were observed. The breakdown voltage was varied between 2 and $6 \mathrm{kV}$, and the currents peaked in the 150 to $450 \mathrm{~A}$ range. These high current values are the consequence of the high capacitance and 


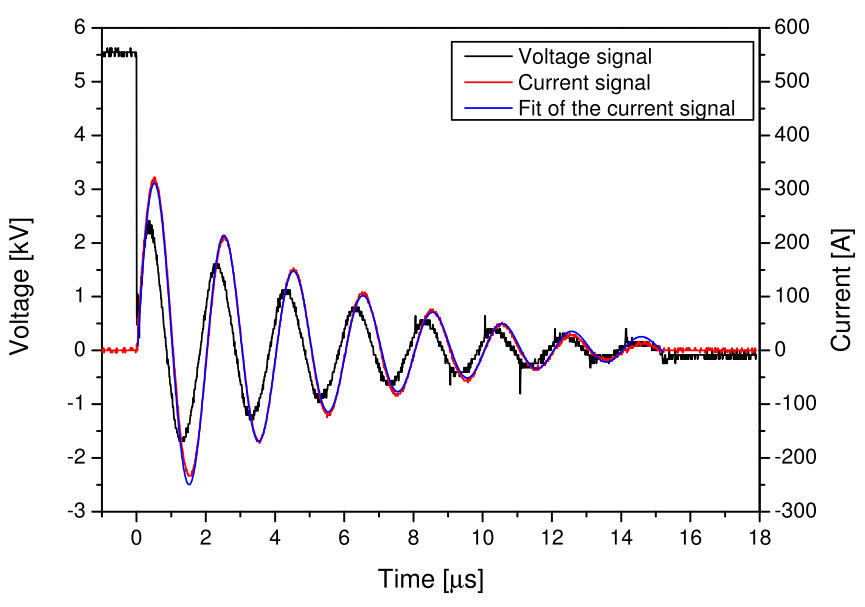

FIG. 3. Voltage (black), and measured (red), and fitted (blue) current signals for a spark ignited between flat-end $\mathrm{Cu}$ electrodes, placed $3 \mathrm{~mm}$ apart.

low resistivity of the circuit, which are needed for the efficient erosion of the electrodes for NP production.

As the simplest approximation, the discharge loop can be represented as a serial RLC circuit. Let us assume that the spark channel is electrically well described by a resistor, $R$, and all the other resistances in the discharge circuit are negligible compared to $R$. This resistance is considered to be connected in series with the rest of the discharge circuit, characterized by an equivalent inductance, $L$, and a capacitance, $C$ (the latter of which is determined by the capacitor). Due to the underdamped behavior of the system (cf. Fig. 3), the $R<2\left(\frac{L}{C}\right)^{0.5}$ criterion is held, and the current waveform can be described by the following equation:

$$
I(t)=I_{0} e^{-\frac{t}{\tau}} \cos \left(\omega t+\varphi_{0}\right),
$$

where

$$
\tau=\frac{2 L}{R} \quad \text { and } \quad \omega=\sqrt{\frac{1}{L C}-\frac{R^{2}}{4 L^{2}}} .
$$

We estimate the total resistance and inductance of the system by fitting this $I(t)$ function to the measured current waveforms (see Fig. 3). This fitting procedure was carried out on all current waveforms collected and it resulted in resistance $(R)$ values in the range of 1.30-1.65 $\Omega$ and an average inductance $(L)$ of $3.6 \mu \mathrm{H}$.

It is expected and also documented in the literature ${ }^{11}$ that the resistance of a spark discharge changes rapidly during its streamer phase, due to the coupled effect of the varying electron density and gas temperature. It should be noted though, that our measurements were done during the conductive phase (i.e., after the streamer electrically connects the two electrodes), in which the spark behaves similar to an arc discharge. ${ }^{23}$ Since the resistance of a pulsed arc also changes slightly, ${ }^{24}$ we attempted to elucidate the time dependence of the $R$ and $L$ quantities by fitting portions of the entire underdamped oscillations and also by using time-dependent $R$ and $L$ values in the fitting process. On one hand, we have not seen systematic variation of these fit parameters within the investigated time range. On the other hand, the above simple electrical model, with its assumption of a constant capacitance and inductance, fits neatly the measured current waveforms, which is in accordance with the findings of Greasom. ${ }^{25}$

The spark discharge was shown to have an inductive component on the order of $10 \mathrm{nH}^{26}$ Since this value is more than 2 orders of magnitude lower than the total inductance of our discharge circuit, the inductance of the discharge gap was neglected with respect to the total inductance of our discharge circuit. More complete electrical models take into account the capacitance of the electrodes and the inductance of the plasma, along with the capacitance, resistance, and inductance of the used probes. ${ }^{18}$ However, as it was mentioned before, the large capacitance of the capacitor bank and inductance of the cables justify our above presented simplifications during the high current conductive phase of the spark discharge.

For calculating the energy dissipated in the spark discharge, one can use the instantaneous power, defined as

$$
P(t)=U(t) I(t)=I^{2}(t) R
$$

and integrate it with respect to time

$$
E(w)=\int_{0}^{w} P(t) d t,
$$

where $w$ defines the time until which one is interested in the energy dissipated in the spark gap. If $w \rightarrow \infty$, one may obtain the total time-integrated spark energy, or spark energy for short.

In most of other studies, the spark energy is estimated by the energy stored in the fully charged capacitor right before the start of the discharge (stored energy), i.e., by using the formula $E=\frac{C V_{b d}^{2}}{2}$ (where $V_{b d}$ is the breakdown voltage and $C$ is the capacitance). However, not all energy stored in the capacitor is dissipated in the spark gap, and therefore, the stored energy is always an upper limit of the spark energy.

We derived the instantaneous power by using the calculated electrical resistance and the measured current waveform. An alternative way of deriving the instantaneous power could go via calculating the product of the voltage and current signals. However, this would require an accurate measurement of the phase shift between the current and voltage signals obtained by the two probes, since a very small phase shift could cause a large error in the calculated power and the resultant energy. Instead, the proposed procedure does not require the accurate measurement of this phase shift and uses only the current waveform for the derivation of the equivalent resistance and the dissipated energy.

As can be seen in Fig. 4(a), the spark energy is smaller than the stored energy. In the particular example shown, the spark energy is only $78 \%$ of the stored energy. For the range of experimental settings used in this study, the spark energy calculation resulted in values between 60 and $430 \mathrm{~mJ}$, equivalent to $78 \%-85 \%$ of the stored energy. These data are shown in Fig. 4(b), as a function of gap length for both electrode geometries. The difference between the stored and spark energies might be due to effects like parasitic losses in the discharge circuit, but outside the spark gap. It is also possible that some energy is dissipated in the spark gap during the postdischarge phase or that the capacitor bank is not fully discharged during the spark. We have also detected that the 

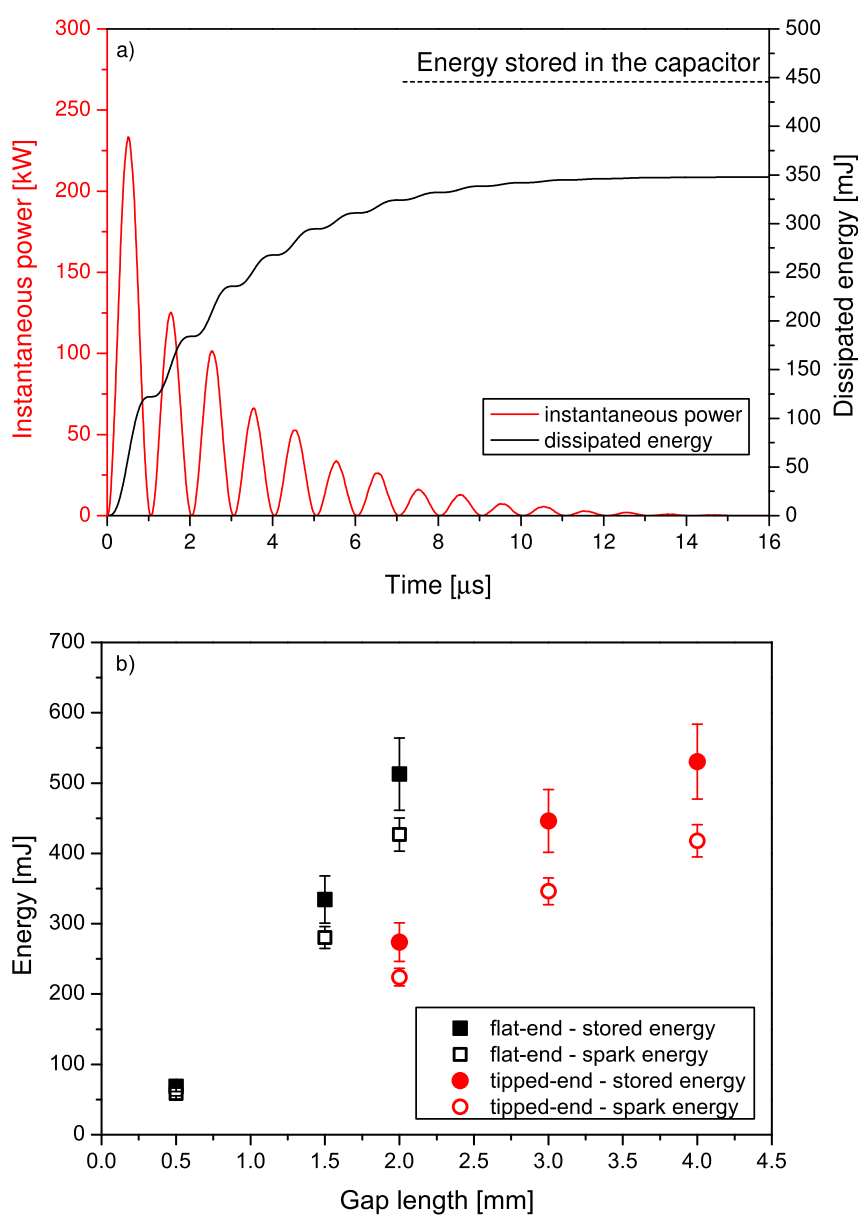

FIG. 4. (a) The instantaneous power (red) and the dissipated energy (black) in the spark gap, calculated from the $\mathrm{I}(\mathrm{t})$ waveform recorded between flatend electrodes $3 \mathrm{~mm}$ apart. (b) The effect of electrode geometry on the energy stored in the capacitor and dissipated in the spark gap as a function of electrode distance.

circuit emits strong electromagnetic radiation, although this particular process is not expected to have a large contribution in the total energy.

\section{B. Imaging results}

For every experimental setting, we performed timeresolved imaging of the spark discharge. The ultimate goal was to shed light on how the discharge morphology and emission intensity evolves over time. In particular, we focused on the time evolution of three parameters, namely, the total light intensity emitted from the spark gap, the axial position of the hottest part, and the average width of the plasma within the discharge channel between the electrodes. We have studied the effect of the gas flow rate, gap size, and electrode geometry on these parameters. In Sec. III B 1, we will describe the overall tendencies obtained, while in Secs. III B 2 and III B 3 we will present the detailed dependencies observed.

\section{General trends and role of electrode geometry}

Fig. 5 illustrates the evolution of spark morphology over time and also allows for the comparison of the effect of the electrode geometry on plasma morphology. Please note that every image shown in Fig. 5 was re-scaled in intensity, in order to make the shape of the discharge discernible also at longer delays, when the emitted intensity is very weak. The images prove that the discharge starts as a thin and intense cylindrical channel between the two electrodes in the case of the tipped-end geometry. As more power is dissipated, the channel diameter as well as the intensity of emission increases, as will be shown later in Fig. 6. After about $500 \mathrm{~ns}$, the channel starts to partially lose its cylindrical shape, but it is still relatively well confined as a quasicylindrical volume between the two electrode tips. By about $10 \mu \mathrm{s}$, when the current oscillations and consequently the energy input cease, the discharge spreads (out) greatly and loses its cylindrical shape almost completely. After this point, the decaying plasma expands in $3 \mathrm{D}$ and gradually fades away.

For flat-end electrodes, the initial situation is very similar, namely, a thin, intense channel grows until its intensity reaches a maximum (cf. Fig. 6). After this point, the shape of the discharge becomes less defined, and loses the cylindrical shape more rapidly than the one generated between tippedend electrodes. The acquired snapshots also prove that in contrast to the tipped case, the spark (channel) wanders over the flat-end electrode surface. It should be noted that we observed a weak afterglow in the millisecond time regime for both geometries, which is in agreement with the strong
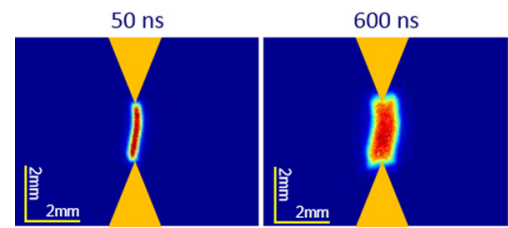

$50 \mathrm{~ns}$

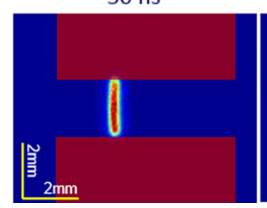

$600 \mathrm{~ns}$
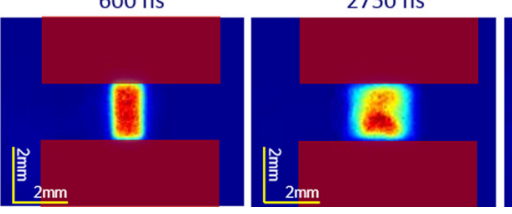

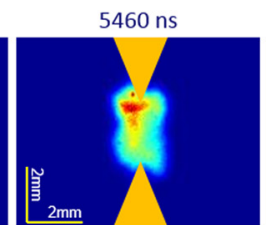

$6550 \mathrm{~ns}$

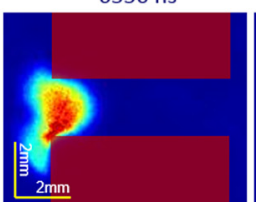

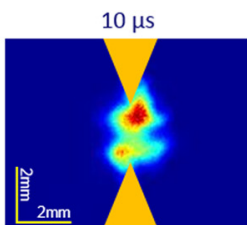

$13.5 \mu \mathrm{s}$

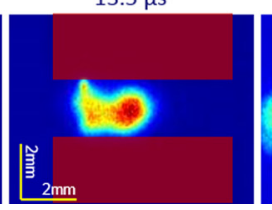

$100 \mu \mathrm{s}$

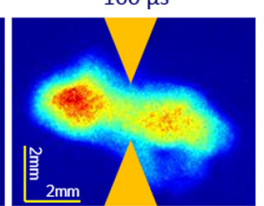

$105 \mu \mathrm{s}$

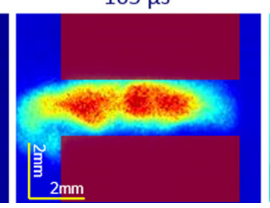

$1 \mathrm{~ms}$

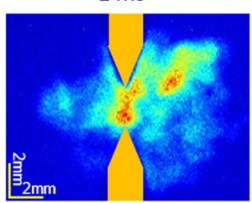

$2.2 \mathrm{~ms}$

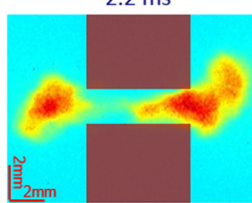

FIG. 5. Images acquired at different delays for spark discharges between tipped-end (upper row) and flat-end (lower row) electrodes placed at 2 mm distance in $10 \mathrm{slm}$ of co-axial $\mathrm{N}_{2}$ flow. The spark energy was 224 and $427 \mathrm{~mJ}$ for the tipped- and flat-end electrodes, respectively. The top electrode was initially the anode in both cases. 


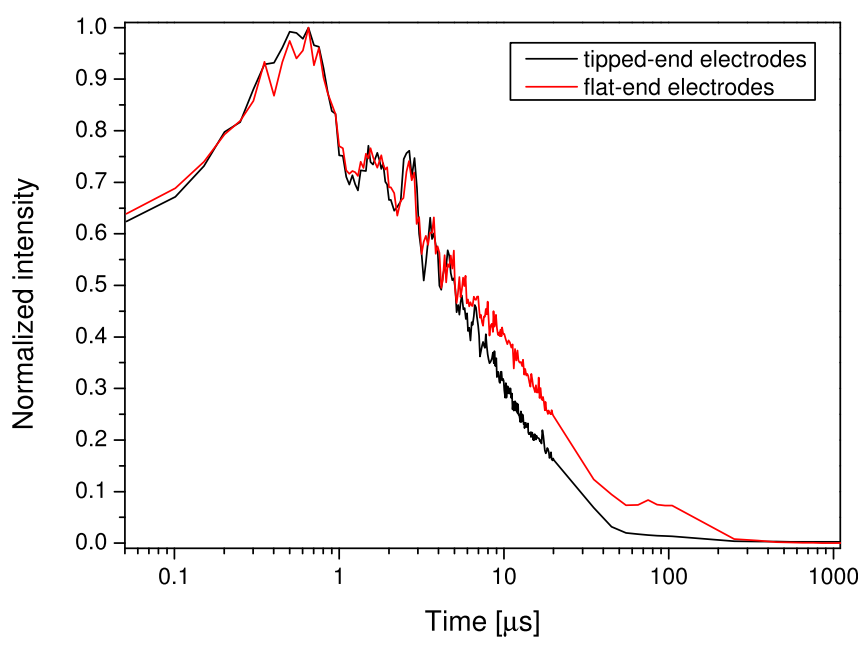

FIG. 6. Total light intensity collected for spark discharges between tippedend (black line) and flat-end (red line) electrodes with a $2 \mathrm{~mm}$ gap. The spark energy is $224 \mathrm{~mJ}$ and $427 \mathrm{~mJ}$ for the tipped and flat geometry, respectively.

"memory effect" observed in the breakdown voltage as a function of spark frequency. ${ }^{27,28}$

We must clarify again that due to the intensity rescaling, the images presented in Fig. 5 only show the shape and the expansion of the emitting vapor at different times. In order to compare the intensity of each image, all the light collected in one frame is integrated and normalized for the different exposure times. In Fig. 6, the total light intensity collected is shown as a function of delay time for sparks established between tipped- and flat-end $\mathrm{Cu}$ electrodes placed at a distance of $2 \mathrm{~mm}$ in a co-axial $\mathrm{N}_{2}$ gas flow of $10 \mathrm{slm}$. The spark energy in the capacitor was 224 and $427 \mathrm{~mJ}$ for the tippedand flat-end geometries, respectively. In order to facilitate comparison, the intensity curves were normalized to their respective maximum. The figure shows how the total light intensity of the spark rises during the first half microsecond for both electrode geometries and then it decays quasiexponentially until a few tens of microseconds. It should be noted that the decay is somewhat slower for the flat-end electrode geometry. After about $50 \mu \mathrm{s}$, the decaying trend changes to a less steep one. In both cases, the light intensity exhibits oscillations in the $1-10 \mu$ s time window, due to the oscillatory nature of the discharge. This also suggests that the light emission at early times is dominated by the main discharge species (i.e., nitrogen species), whereas at later times, more and more contribution comes from the excitation of copper vapor accumulating in the spark gap due to electrode erosion. Although it cannot be excluded that any change in the total light intensity is partially due to a change of the main emission wavelength (our detector has peak sensitivity at around $520 \mathrm{~nm}$ ), it is safe to assume that, at least for the decaying part of the light intensity curve, the decreasing tendency is due to the decreasing plasma temperature caused by the decrease in the instantaneous electrical power of the discharge.

The snapshots taken for tipped electrode geometry and shown in Fig. 5 exhibit another striking feature of the spark plasma. The plasma not only changes in intensity and shape over time, but the recorded snapshots also reveal an uneven intensity distribution along the axis of the electrodes. For example, in the frames shown in Fig. 5, there is a "plasma hot spot" (i.e., a spot with distinctively more intense emission) in front of the lower electrode at $2260 \mathrm{~ns}$, then in front of the upper electrode at $5460 \mathrm{~ns}$, and in front of the upper electrode again at $10 \mu \mathrm{s}$. This behavior was also detected for flat-end electrodes, but to a lesser extent. Note that the existence of such a hot spot does not mean that the discharge would not fill the entire gap. Fig. 7 illustrates how the position of this "plasma hot spot" wanders in the gap for both electrode geometries. Initially, that is, at delays smaller than about $1 \mu \mathrm{s}$, the plasma seems to be evenly distributed in the gap. Later, typically in the $1-12 \mu$ s time range, the position of the "plasma hot spot" oscillates between the electrodes. For the flat geometry, some displacement of the plasma hot spot position between the electrodes can also be detected. At delays longer than $15 \mu \mathrm{s}$, the position of the maximum emission intensity settles in the center of the spark gap independently of the electrode geometry.

We also studied how the width of the spark channel evolves in time. The average channel width was determined by averaging the full width at half maximum of the light intensity traces perpendicular to the electrode axis for every row of the ICCD-image located between the two electrodes. Fig. 8 depicts the temporal evolution of the channel width for up to $20 \mu \mathrm{s}$. Note that the determination of the channel width is complicated by the existence and axial movement of the plasma hot spot and the gradual loss of the initially welldefined cylindrical shape of the channel, which is well reflected in the degraded accuracy of channel width values at delays beyond about $3.5 \mu \mathrm{s}$. Still, the following three tendencies are clear and conclusive. First, the discharge sets off as a narrow channel (its width is below $500 \mu \mathrm{m}$ ) and then it grows monotonically, in both geometries. Second, for flatend electrodes, a steeper initial rise of the average channel width was obtained. Last, the channel width stabilizes

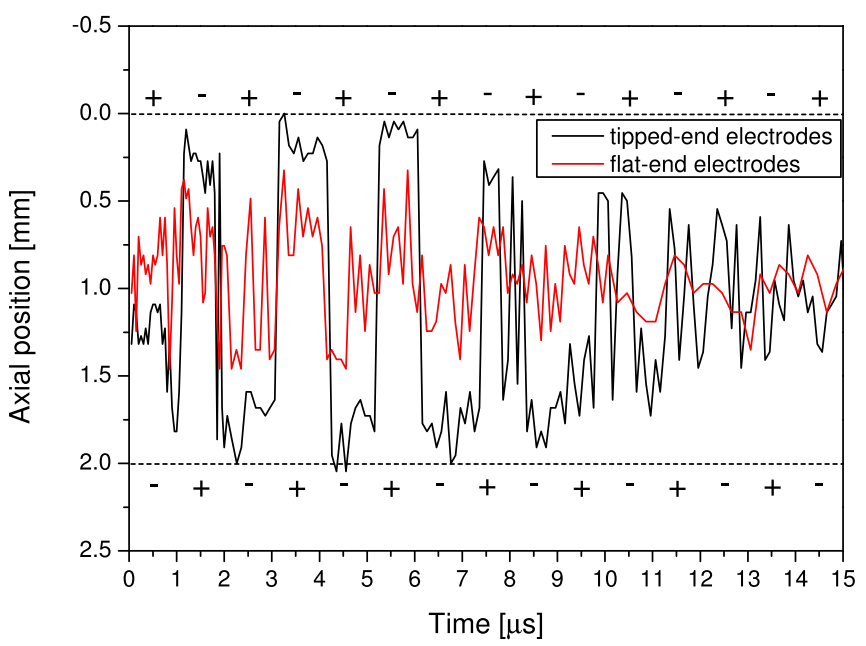

FIG. 7. Axial position of "plasma hot spot" for oscillatory spark discharges between tipped-end (black line) and flat-end (red line) electrodes placed $2 \mathrm{~mm}$ apart. The spark energy was 224 and $427 \mathrm{~mJ}$ for the tipped and flat geometry, respectively. The positions of the electrodes are marked with dashed lines. The electrode polarity is also shown. 


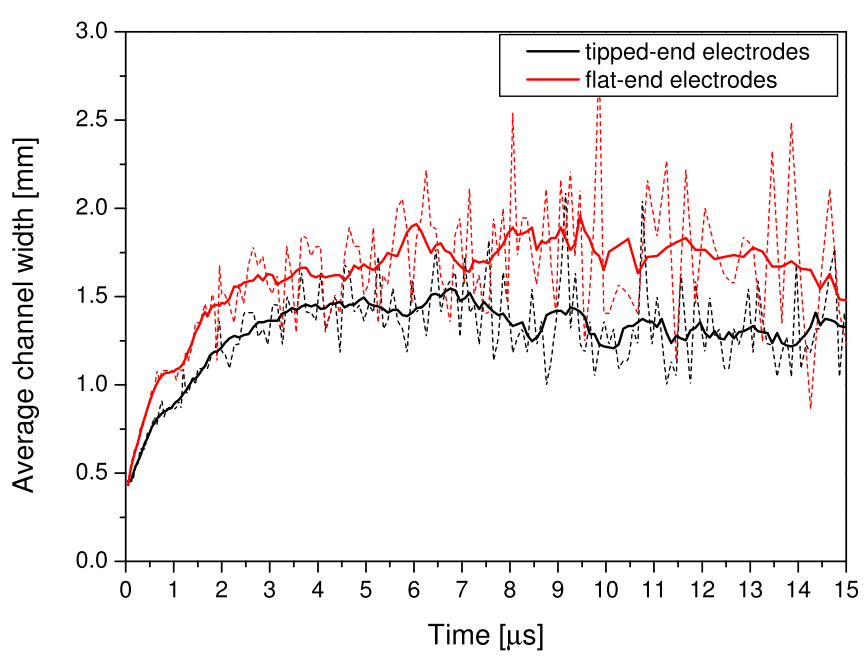

FIG. 8. Average channel width for spark discharges between tipped-end (black line) and flat-end (red line) electrodes at a $2 \mathrm{~mm}$ distance. The spark energy was 224 and $427 \mathrm{~mJ}$ for the tipped and flat geometry, respectively. The dotted lines show the measured values of the channel widths, while the solid lines are obtained by arithmetic averaging (using a 9-point moving window).

around a value of about $1.5 \mathrm{~mm}$ for both electrode geometries (within measurement error).

\section{Effect of gap size}

In this section, we summarize our observations related to the effect of the gap size on the studied spark parameters, as were obtained from our imaging measurements. Fig. 9 presents the time-resolved total light intensity curves for the two electrode geometries. The general trend is that the total light intensity emitted is higher for larger gaps for both electrode geometries, which is in line with the observations that with increasing electrode distance, the energy dissipated in the gap is also increasing. We note that this trend is more pronounced for the flat geometry.

The variation of the axial position of the "plasma hot spot" with the gap length is depicted in Fig. 10. For the tipped electrode geometry (Fig. 10(a)), the results clearly show oscillations between the electrodes, which become more pronounced with increasing gap sizes. Despite the measurement noise, it can also be clearly seen how the plasma tends to settle at the center of the gap as the oscillations decay. For the flat-end geometry (Fig. 10(b)), the situation is markedly different. The periodic movement of the plasma hot spot between the flat-end electrodes is hardly visible from these results (the sole exemption is the first $1-8 \mu$ s portion of the $2 \mathrm{~mm}$ gap data set). The plasma hot spot practically stays in the center of the gap for the whole duration of the discharge.

Finally, Fig. 11 shows the evolution of average channel width over time for several gaps for both geometries. As can be seen, the spark channel undergoes similar expansion and becomes slightly wider at larger gaps for both geometries. After reaching a maximum, the channel width slowly moves towards values around $1.5 \mathrm{~mm}$, for all gaps. Similarly to the tendency in the hot spot position, the case of $0.5 \mathrm{~mm}$ gap in the flat-end electrode geometry stands out; the average channel width is smaller, and it exhibits considerably less variation in time.
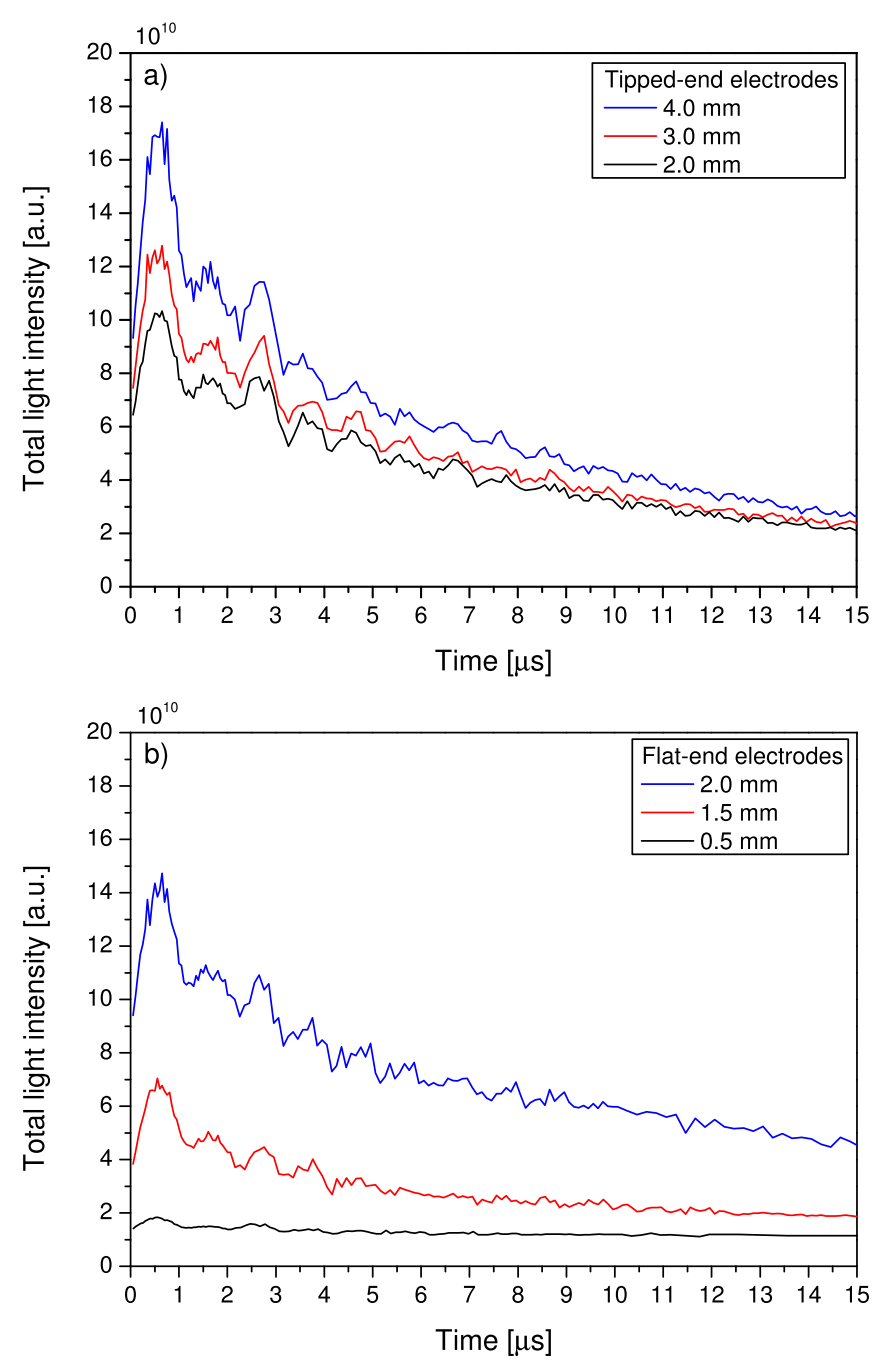

FIG. 9. The temporal evolution of total emitted light intensity collected for (a) tipped-end and (b) flat-end electrodes placed at different distances.

\section{Effect of gas flow rate}

In order to study the effect of gas flow rate on the emission and geometry of the spark discharge, time-resolved plasma morphology measurements were carried out for co-axial nitrogen flows of $10 \mathrm{slm}, 8 \mathrm{slm}, 6 \mathrm{slm}, 4 \mathrm{slm}$, and $1.7 \mathrm{slm}$. Tipped-end electrodes were used with a $3 \mathrm{~mm}$ gap size, and the spark energy was kept around $200 \mathrm{~mJ}$. The results reveal that in the flow range examined the gas flow rate has no significant influence on the time evolution of the morphology and light emission of the spark plasma. The total light intensity collected, the plasma hot spot position, and the channel width exhibited the very same behavior for all the flows used; thus, the curves obtained were identical to those shown in Figures 6-11.

\section{DISCUSSION}

\section{A. The correlation of electrical and imaging data}

As it was alluded to already, the total light intensity exhibits oscillations over time. Fig. 12(a) shows that these oscillations neatly follow the oscillations of the instantaneous power. ${ }^{29,30}$ It must be noted that the instantaneous power 

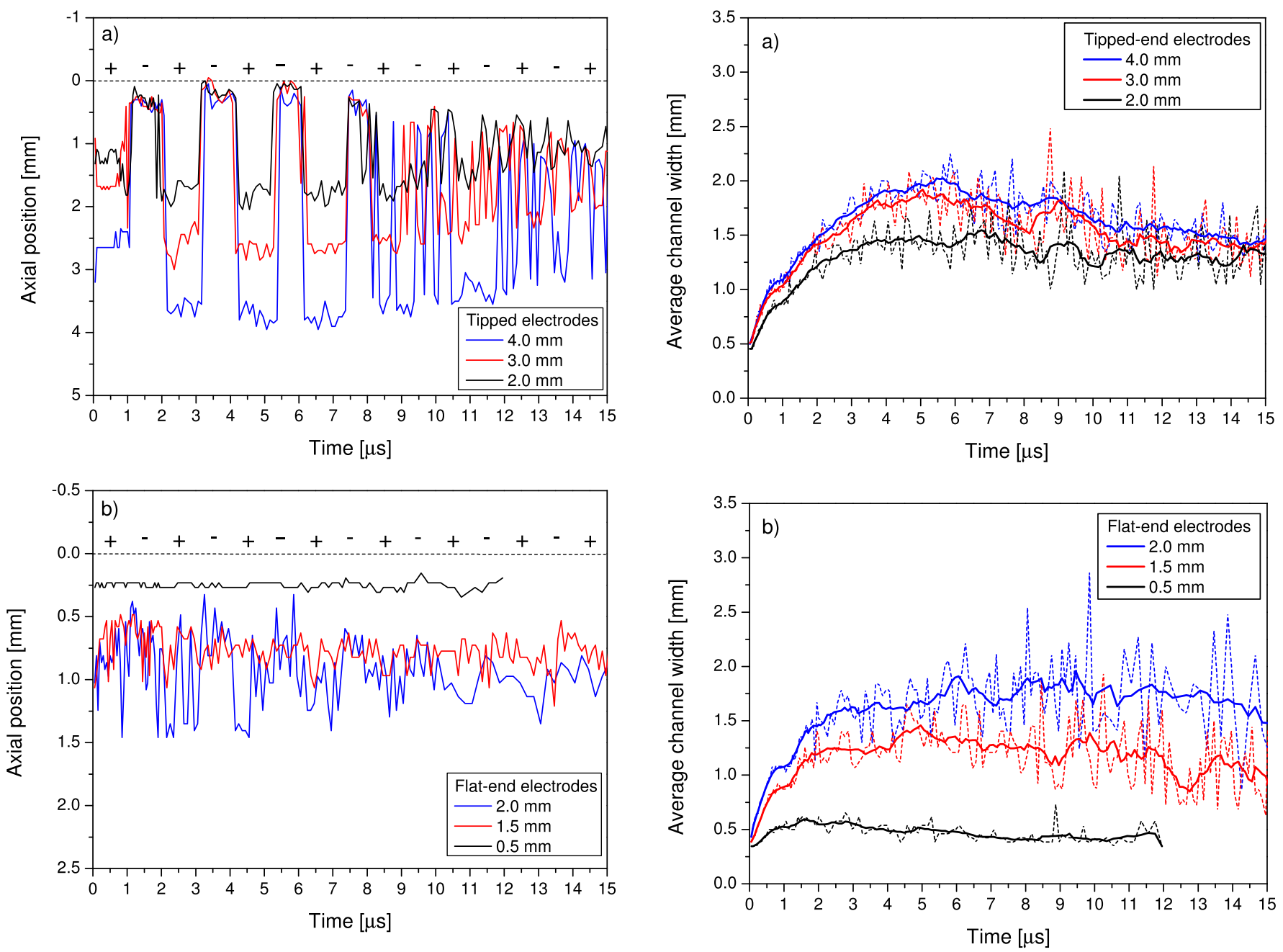

FIG. 10. The temporal evolution of the axial position of the most luminous part (plasma hot spot) of the plasma for (a) tipped-end and (b) flat-end electrode geometries at different gap lengths. Note that for the sake of clarity, only the position of one electrode is shown given by the dotted line, for reference. The electrode polarity is also shown.

is calculated as $I^{2}(t) R$, where $I(t)$ is the current measured in the discharge circuit and $R$ is the equivalent resistance of the spark gap, which was calculated to be $1.4 \Omega$ in our SDG in the particular case shown below (see Section III A).

Fig. 12(b) shows how the position of the plasma hot spot oscillates over time and how it correlates with the current waveform. As is known, the current exhibits an oscillatory behavior, meaning that the electrode polarity alternates typically less than 20 times in the $0-20 \mu$ s temporal window, i.e., anode and cathode reverses several times during the lifetime of a spark. It can also be seen that the displacement of the plasma hot spot becomes smaller as time passes, i.e., as the strength of the electric field is decreasing. Note that these findings hold for every other experimental parameters (e.g., gap sizes, flow rates, etc.), and the data shown in Fig. 12 only exemplify the tendencies by displaying the case of tipped electrodes placed at a $3 \mathrm{~mm}$ distance.

Data in Fig. 12(b) prove that the plasma hot spot is always situated close to the momentary negative electrode (cathode) and the position of the most luminous part of the discharge follows the periodicity of the current signal with some delay. The axial structure of the discharge channel

FIG. 11. Time evolution of the channel width for (a) tipped-end and (b) flatend electrode geometries, at three different gap lengths. The dotted lines show the measured values of the channel widths, while the solid lines are obtained by arithmetic averaging (using a 9-point moving window).

seems to be similar to that of a glow discharge ${ }^{31}$ or a DC continuous arc as defined by Boumans. ${ }^{23}$ Close to the (momentary) negative electrode, a region with non-zero charge, known as the "cathode fall," forms in the gap, which effectively shields the electric field. When moving beyond the cathode fall region, i.e., a bit further towards the anode, the plasma tends to be more intense in emission due to the dissipation of a substantial amount of energy gained by the electrons in the fall region. We suppose that this latter process causes the formation of the most luminous plasma region in the spark gap, hereby referred to as "plasma hot spot." When polarity changes, the existing hot spot collapses fast and another one builds up around the opposite electrode, which turns to be the instantaneous cathode during the next half cycle of the oscillations. It should be noted that our present imaging setup cannot spatially resolve the cathode fall region in the vicinity of the momentary cathode, but the behavior of the discharge is fully in line with the above given arc-like behavior and hence provides an indirect evidence on the existence of the cathode fall region during the spark discharge.

Tipped electrodes produce stronger electric fields which assist in the localization of the "plasma hot spot" and 

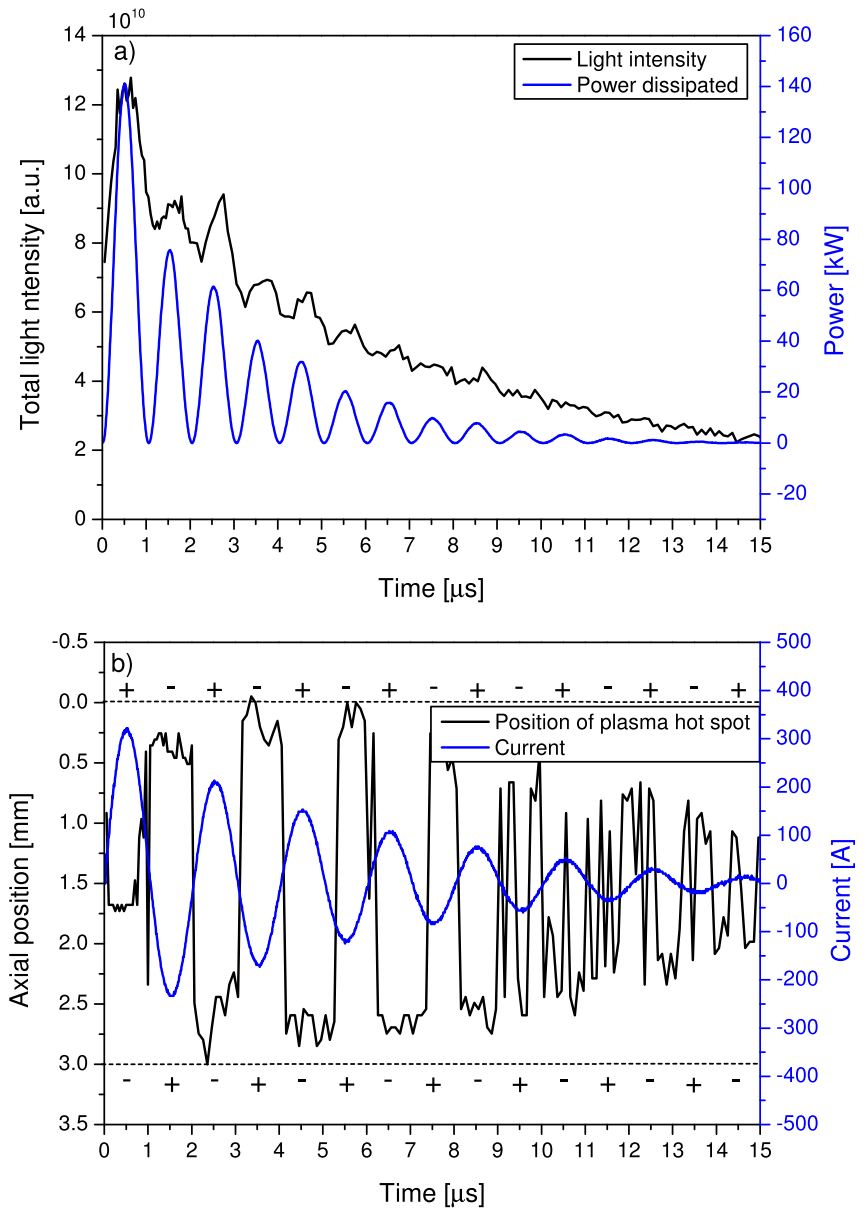

FIG. 12. Imaging results compared to electric signals (tipped-end electrodes, $3 \mathrm{~mm}$ gap length). (a) Total light intensity collected (black line) compared to instantaneous power (blue line). (b) Axial position of the plasma hot spot (black line) compared to the current waveform (blue line). The positions of the electrodes are marked with dashed lines. The electrode polarity is also shown.

stabilize its oscillation according to the voltage polarity. As opposed to this, the weaker electrical field between the flat electrodes cannot induce an apparent intense plasma spot, and consequently, the plasma hot spot oscillation is not pronounced, either (Figs. 7 and 10(b)). It should be mentioned that the oscillation frequency of the axial position of the hot spot observed in Fig. 12(b) is half of the oscillation frequency of the emitted intensity shown in Fig. 12(a). This is so because the periodicity of the emitted light follows the instantaneous power, which is proportional to the square of the current.

\section{B. Channel expansion}

The expansion velocity of the plasma front can be determined using the slope of the average channel radius vs. time curve (also considering that the radial distance of the external front of the channel from the channel axis is the half of the average channel width). In Fig. 13, we re-plotted the initial part of the average channel width curve from Fig. 8 at a gap length of $4 \mathrm{~mm}$. The figure indicates that in the initial phase of the discharge, up until about $500 \mathrm{~ns}$ after the breakdown, the expansion velocity of the plasma front is nearly
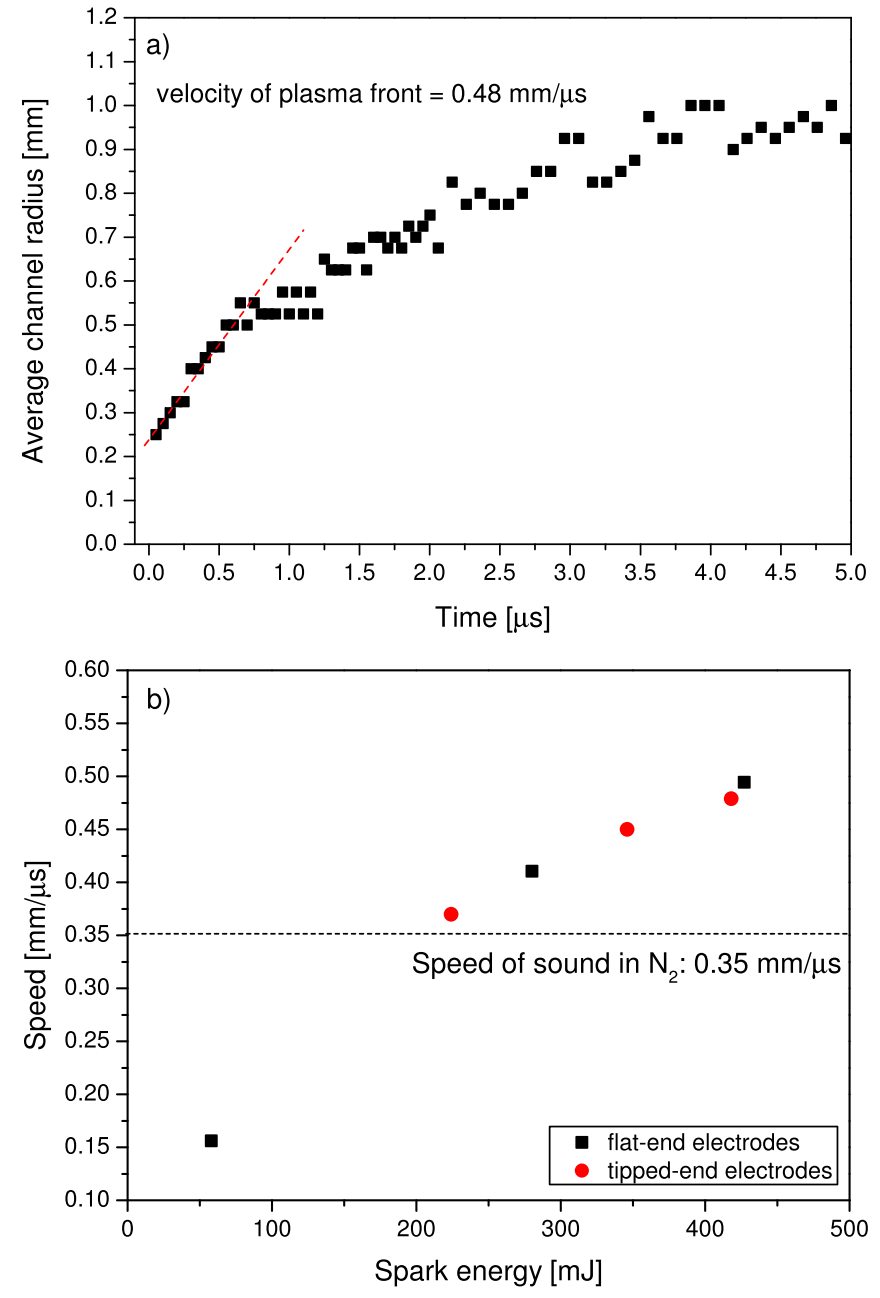

FIG. 13. (a) Temporal evolution of the average channel radius for a spark discharge maintained between tipped electrodes at $4 \mathrm{~mm}$ gap length. The dashed red line indicates an initial plasma front expansion velocity of $0.48 \mathrm{~mm} / \mu \mathrm{s}$. (b) Initial expansion velocity of the plasma front as a function of the spark energy. Results for tipped electrodes (red circles) are presented together with flat-end ones (black squares). The dotted line marks the speed of sound in nitrogen at room temperature.

constant and equals to about $0.48 \mathrm{~mm} / \mu \mathrm{s}$. This value is higher than the speed of sound in $\mathrm{N}_{2}$ at room temperature, which is $0.35 \mathrm{~mm} / \mu \mathrm{s}$. This means that the expansion of the spark channel, as derived from the average channel radius values, starts at supersonic speeds and slows down afterwards. Such fast growth of the plasma channel, together with the inherent change in temperature and gas pressure induces a shockwave. ${ }^{32}$ The supersonic expansion was confirmed directly, for all but one cases used in this work, since the characteristic shockwave sound was clearly audible during sparking. One can add that the gas temperature in the gap before the spark may be higher than room temperature as a result of earlier spark events. If this is the case, the speed of sound is somewhat higher than $0.35 \mathrm{~mm} / \mu$ s according to the Newton-Laplace equation.

It is plausible to assume that at the beginning of the expansion, the radii of the spark channel and the shockwave front coincide. ${ }^{33}$ Therefore, we can take our measurements of the initial front speed as a measurement of the shockwave velocity. Reinmann and $\mathrm{Akram}^{34}$ used an interferometric method to follow the shockwaves created in similar spark 
discharges. They obtained radial velocities of the shockwave of about $0.6 \mathrm{~mm} / \mu \mathrm{s}$, which is somewhat faster than the highest expansion velocity we obtained. This difference can be explained by the different currents in the two cases. In the experiments reported by Reinmann and Akram, the current raised to about $400 \mathrm{~A}$ in less than $100 \mathrm{~ns}$, while in our setup, the rate of current increase was smaller: it took about $500 \mathrm{~ns}$ to reach a value around $260 \mathrm{~A}$.

From the curves shown in Fig. 11, we can also obtain the initial expansion velocity of the plasma front (or the speed of the shockwave) at different gap lengths in both electrode geometries. In Fig. 13(b), the initial expansion velocity, characteristic to approximately the first $500 \mathrm{~ns}$ after the breakdown, is plotted as a function of spark energy. The results indicate that the initial speed of the plasma front increases with gap length for both tipped- and flat-end electrode geometries. The graph convincingly proves that the plasma channel expansion scales quasi-linearly with spark energy for all electrode conditions used in the present study, and that this velocity is higher than the speed of sound, except for the $0.5 \mathrm{~mm}$ gap length. As mentioned before, flatend electrodes placed at $0.5 \mathrm{~mm}$ distance show a different behavior, most probably because under that condition the discharge is working in the micro-discharge regime..$^{35,36}$ Since the spark energy can be controlled by external parameters such as the spark gap distance, electrode geometry, and circuit capacitance, it is then possible to adjust or control the expansion velocity of the spark channel by tuning these parameters.

As it is obvious in Fig. 13(a), the plasma front slows down after its initially fast expansion. At this moment, the shockwave is assumed to continue its fast propagation, thereby leaving the plasma channel behind. After this point ( $\sim 750 \mathrm{~ns}$ after the onset of the breakdown), the behavior of the spark channel can be described by adopting physical models originally developed for arc discharges. Braginskii ${ }^{33}$ calculated the expansion of the arc radius right after its ignition (i.e., during the "spark phase") using a hydrodynamic model and solving the energy balance equation, assuming no thermal or radiative losses and a constant electron temperature. He found that the plasma radius $r$ is proportional to $I^{1 / 3} t^{1 / 2}$, where $I$ and $t$ represents the current passing through the gap and the time, respectively. In Fig. 14, we show how the average channel radius compares to this function of Braginskii. In order to facilitate the comparison of the temporal behavior, we incorporated a scaling factor in the expression to provide similar width values. The curves in Fig. 14 show that the channel radius of our spark discharge has a temporal trend similar to that of an arc discharge.

Another similarity with an arc is the value of the discharge's equivalent electrical resistance. Different time dependent calculations of the arc's electrical resistance, based on the temporal evolution of the current, can be found in the scientific literature. Because of their relative simplicity, we took the expression given in Ref. 37 (based on a model developed by Rompe and Weizel) as an example and the one calculated by Barannik et al. ${ }^{24}$ Although these expressions were meant for unipolar current peaks with higher intensity (up to several kiloamps), one can use them to estimate the

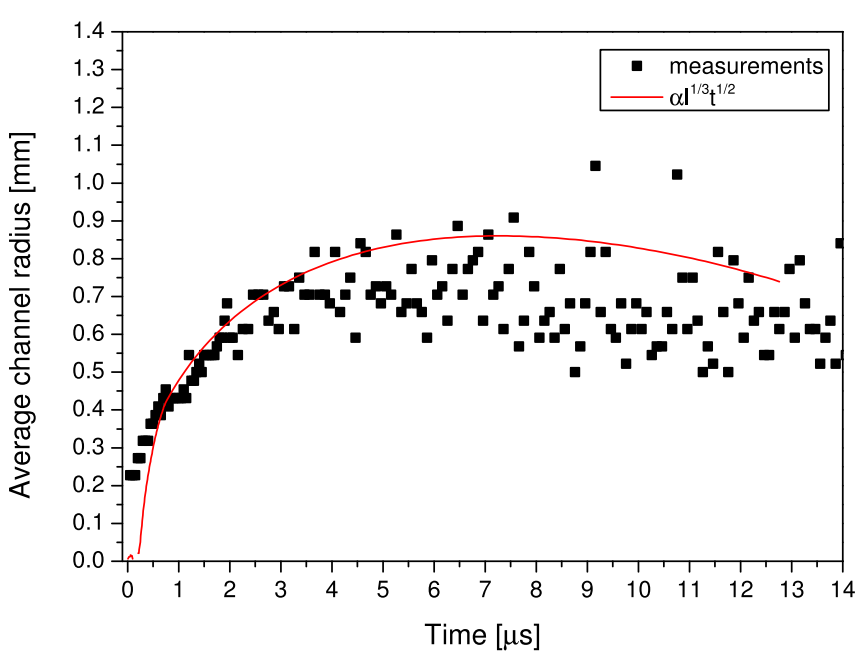

FIG. 14. Temporal evolution of the average channel width of a spark discharge (tipped electrodes, $2 \mathrm{~mm}$ gap) compared to the evolution of the channel radius calculated by the formula given in Ref. 33 (red line).

resistance at the first peak of the current waveform $(\sim 0.5 \mu \mathrm{s})$. These expressions give resistances in the range of $0.9-1.3 \Omega$ (Ref. 37) and 1.3-1.9 $\Omega$ (Ref. 24) for the conditions studied in the present work. In Section III B 1, the plasma (spark gap) resistance was calculated to be in the range of $1.3 \Omega-1.65 \Omega$ for the conditions in our SDG. These values match fairly well the arc resistances estimated by the above models.

\section{CONCLUSIONS}

In the present work, we have performed a timeresolved imaging and electrical study of a spark discharge generator in the range of typical experimental conditions used for nanoparticle synthesis. We studied the effect of several experimental parameters (electrode geometry, electrode gap, and gas flow rate) on the temporal evolution of plasma morphology and electrical discharge characteristics. The imaging and electrical data recorded were also correlated to each other and evaluated together. Our results and findings provide information for the understanding of the working principles of SDGs and are expected to be useful in establishing spectroscopy-based monitoring of SDG operation.

We found that a series RLC circuit decently describes the temporal behavior of the discharge circuit, i.e., the spark is an under-damped oscillatory discharge after the breakdown. Although the discharge channel might have some minor inductance and capacitance, these are negligible compared to the total capacitance and inductance of the discharge circuit; therefore, the plasma channel was considered as a purely resistive load. The good fit obtained between the measured current and the modeled one indicates the plasma channel is well approximated by a constant resistive load connected in series with the circuit's capacitance and inductance. A comparison of the oscillations in the total light intensity emitted also shows good agreement with the calculated instantaneous power dissipated on the spark's equivalent resistance. 
We have determined the energy stored in the capacitor and the energy dissipated during the course of the spark discharge. We also observed a difference between these two energies. This difference might be due to an incomplete discharge of the capacitor or to parasitic losses of the discharge circuit (backward currents toward the power supply). It is also possible that some energy is dissipated in the postdischarge phase, which is supported by our imaging results, indicating weak emission of the spark gap (i.e., a kind of afterglow) up to milliseconds after the current oscillations came to an end.

Our results obtained on the temporal evolution of the plasma morphology clearly proves i) the fast radial expansion of the plasma channel after breakdown, ii) the development of an axial emission inhomogeneity in the gap in the $1-15 \mu \mathrm{s}$ time domain, and iii) the oscillation of this plasma hot spot between the electrodes. We showed that these plasma oscillations follow the polarity reversal of the electrodes during the oscillatory discharge and that the plasma tends to be more luminous close to the momentary negative electrode (cathode). This effect has important implication to NP generation since it confirms that both electrodes are eroded by the discharge, possibly to a somewhat different extent (depending on the actual electrical parameters of the discharge circuit). We measured that the plasma channel expands at supersonic speeds during the first, less than $1 \mu \mathrm{s}$ long stage after breakdown, which indicates the formation of a shockwave. This initial expansion speed scales linearly with spark energy, which opens the possibility of controlling the channel expansion by adjusting the spark energy.

Finally, the expansion of the spark channel is proven to follow a similar behavior of that of a transient arc after ignition. Similarly, the averaged values of spark resistance are in agreement with those obtained for transient arc discharges. These findings seem to indicate that the spark discharge can be considered to be in the arc regime after the breakdown and for a few microseconds afterwards, until the current oscillations stops. However, our results are compared with calculations for the expansion and resistance of unipolar arcs while we have an oscillatory discharge. It is possible that several spark and arc phases alternate during the current and voltage oscillations. Consequently, our findings only indicate an average similarity with the arc regime. This could have important consequences for the understanding and modeling of the NP generation process in similar SDGs. However, more tests are needed in order to confirm this hypothesis, in particular, the state of thermal equilibrium will be checked by means of temporally and spatially resolved spectroscopy in our ongoing work.

\section{ACKNOWLEDGMENTS}

This project has received funding from the European Union's Seventh Framework Program (FP7/2007-2013) under Grant Agreement No. 280765 (BUONAPART-E) and from the TÁMOP Programme of Hungary (No. 4.2.2.A-11/ 1/KONV-2012-0047).

${ }^{1}$ T. V. Pfeiffer, J. Feng, and A. Schmidt-Ott, Adv. Powder Technol. 25, 56 (2014).

${ }^{2}$ J. H. Byeon, J. H. Park, and J. Hwang, J. Aerosol Sci. 39, 888 (2008).

${ }^{3}$ B. O. Meuller, M. E. Messing, D. L. J. Engberg, A. M. Jansson, L. I. M. Johansson, S. M. Norlén, N. Tureson, and K. Deppert, Aerosol Sci. Technol. 46, 1256 (2012).

${ }^{4}$ E. Hontanon, J. M. Palomares, M. Stein, X. Guo, R. Engeln, H. Nirschl, and F. E. Kruis, J. Nanopart. Res. 15, 1957 (2013).

${ }^{5}$ T. Sugai, H. Omote, S. Bandow, N. Tanaka, and H. Shinohara, J. Chem. Phys. 112, 6000 (2000).

${ }^{6}$ T. Sugai, H. Yoshida, T. Shimada, T. Okazaki, and H. Shinohara, Nano Lett. 3, 769 (2003).

${ }^{7}$ G. Ganteför, H. R. Siekmann, H. O. Lutz, and K. H. Meiwes-Broer, Chem. Phys. Lett. 165, 293 (1990).

${ }^{8}$ N. S. Tabrizi, M. Ullmann, V. A. Vons, U. Lafont, and A. Schmidt-Ott, J. Nanopart. Res. 11, 315 (2009).

${ }^{9}$ T. E. Itina and A. Voloshko, Appl. Phys. B. 113, 473 (2013).

${ }^{10}$ J-P. Borra, J. Phys. D. 39, R19 (2006).

${ }^{11}$ D. Z. Pai, D. A. Lacoste, and C. O. Laux, Plasma Sources Sci. Technol. 19, 065015 (2010).

${ }^{12}$ M. Janda, Z. Machala, A. Niklova, and V. Martisovit, Plasma Sources Sci. Technol. 21, 045006 (2012).

${ }^{13}$ G. Pilla, D. Galley, D. A. Lacoste, F. Lacas, D. Veynante, and C. O. Laux, IEEE Trans. Plasma Sci. 34, 2471 (2006).

${ }^{14}$ Z. Machala, M. Janda, K. Hensel, I. Jedlovsky, L. Lestinska, V. Foltin, V. Martisovits, and M. Morvova, J. Mol. Spectrosc. 243, 194 (2007).

${ }^{15}$ T. Verreycken and P. J. Bruggeman, Plasma Sources Sci. Technol. 23, 015009 (2014).

${ }^{16}$ Z. Machala, I. Jedlovský, and V. Martišovitš, IEEE Trans. Plasma Sci. 36, 918 (2008).

${ }^{17}$ S. Nijdam, C. G. C. Geurts, E. M. van Veldhuizen, and U. Ebert, J. Phys. D: Appl. Phys. 42, 045201 (2009).

${ }^{18}$ M. Janda and Z. Machala, IEEE Trans. Plasma Sci 39, 2246 (2011).

${ }^{19}$ B. Du, S. Mohr, D. Luggenholscher, and U. Czarnetzki, J. Phys. D: Appl. Phys. 44, 125204 (2011).

${ }^{20}$ M. Ramli and K. Wagatsuma, ISIJ Int. 50, 864 (2010).

${ }^{21}$ A. Hamdan, I. Marinov, A. Rousseau, and T. Belmonte, J. Phys. D: Appl. Phys. 47, 055203 (2014).

${ }^{22}$ See http://www.buonapart-e.eu/ for official website with general information on the project members, objectives, results and contact information.

${ }^{23}$ P. W. J. M. Boumans, in Excitation of Spectra in Analytical Emission Spectroscopy, edited by E. L. Grove (Marcel Dekker, New York, 1972).

${ }^{24}$ T. G. Engel, A. L. Donaldson, and M. Kristiansen, EEE Trans. Plasma Sci. 17, 323 (1989).

${ }^{25}$ W. D. Greason, Z. Kucerovsky, S. Bulach, and M. W. Flatley, IEEE Trans. Ind. Appl. 33, 1519 (1997).

${ }^{26} \mathrm{P}$. Persephonis, K. Vlachos, C. Georgiades, and J. Parthenios, J. Appl. Phys. 71, 4755 (1992).

${ }^{27}$ S. Nijdam, G. Wormeester, E. M. Veldhuizen, and U. Ebert, J. Phys. D: Appl. Phys. 44, 455201 (2011).

${ }^{28}$ N. T. Nesic, M. M. Pejovic, and E. N. Zivanovic, J. Phys. D: Appl. Phys. 44, 095203 (2011).

${ }^{29}$ J. Crager and M. Horenstein, Inst. Phys. Conf. Ser. 178, 149 (2003).

${ }^{30}$ J. W. Flowers, Phys. Rev. 64, 225 (1943).

${ }^{31}$ J. M. Meek and J. D. Craggs, Electrical breakdown of Gases (Clarendon Press, Oxford, 1953).

${ }^{32}$ P. Krehl and S. Engemann, Shock Waves 5, 1 (1995).

${ }^{33}$ S. I. Braginskii, Sov. Phys.-JETP 7(6), 1068 (1958).

${ }^{34}$ R. Reinmann and M. Akram, J. Phys. D: Appl. Phys. 30, 1125 (1997).

${ }^{35}$ K. H. Becker, K. H. Schoenbach, and J. G. Eden, J. Phys. D: Appl. Phys. 39, R55 (2006).

${ }^{36}$ D. Mariotti and R. M. Sankaran, J. Phys. D: Appl. Phys. 43, 323001 (2010).

${ }^{37}$ D. Pommerenke, J. Electrostatics 36, 31 (1995). 